\title{
Multimodal imaging in the evaluation of patch test results
}

\author{
by B. Tomaka*, M. Szwedo* and J. Targosz** \\ * MONIT SHM Sp. z o.o., ul. Gromadzka 66, 31-719 Kraków, Poland, b.tomaka@gmail.com \\ ${ }^{*}$ AGH University of Science and Technology, Al. Mickiewicza 30, 30-059 Kraków, Poland
}

\begin{abstract}
Contact allergy diagnostics in many cases is based on subjective techniques of evaluation. The clinical evaluation of patch test reaction is achieved by visual impression combined with measurements of the intensity of the reaction on the usual scale of differentiation, and it is likely that some allergic reactions will not be noticed, because of weak or delayed inflammation. Authors are suggesting a new attitude to that problem with the use of infrared imaging and multimodal imaging methods as a supplementary non-contact technique for more thorough evaluation.
\end{abstract}

\section{Extended Abstract}

Dermatological diagnostic of contact allergy diagnostics is a long and complicated process. It demands careful review of work and environmental history, obtain chronologic exposure histories that include hobbies and specific activities relative to onset of the dermatitis. Patch testing is a method of medical diagnosis that attempts to provoke a small, controlled, allergic response (allergic contact dermatitis, ACD). Although the sensitivity and specificity of patch testing varies according to the allergen, it has been shown to be cost effective if performed early in the course of the disease in patients with chronic ACD by reducing pre-diagnosis costs of treatment. Treated patients confirmed by patch testing exhibit significantly greater improvement in dermatology-specific quality of life than those patients who were not patch tested [1].

Evaluation of skin test reaction to allergen is achieved by visual impression combined with measurements of the intensity of the reaction on the scale of differentiation. Visual assessment does concern only skin surface, so difficulties like changes in skin pigmentation, atopic skin, black race skin or delayed reaction may occur. Common problem is interpretation of lesions as irritant, doubtful and weak reaction, especially at second reading, when uncertain grades are not allowed. That is why it is an non-objective diagnostic method and any methods to support assessment are used.

The purpose of our work is to develop of a method to support the evaluation of patch tests with the use of multimodal imaging based on studies in different ranges of light radiation. The fusion of medical images is the process of registering and combining images from one or many imaging techniques. It relates this to improving the quality of diagnostic information or its complementation in order to increase the clinical usefulness of medical images for diagnosis and their evaluation. Multimodal medical image fusion algorithms support the improvement of the accuracy of clinical decisions taken on the basis of medical images [2].

The essence of the thermography $[3,4,5]$ is to compare temperature of skin with positive reaction to allergen with temperature of surrounding healthy skin, so information about gradient of temperature is more important than its absolute value. Thermography is a valuable, non-contact measurement technique, which can find practical application in dermatological diagnostics, but only if all standardisation rules are adhered. Every negligence in testing procedure may produce an effect that may distort a result and make it unreliable.

Patch test is the only useful and reliable method for identification of an allergic contact dermatitis - it is acknowledged as the gold standard. The International Contact Dermatitis Research Group (ICDRG) was formed to promote the understanding of contact dermatitis and it has had major roles in the standardization of patch testing for over fifty years [6].

Emissivity of biological tissues is within 0.85 and 0.98 . For human skin it is reported to be equal $0.98 \pm 0.01$ for wavelength range of $2-20 \mu \mathrm{m}$, it peaks around $10 \mu \mathrm{m}$, regardless of skin colour. Generally, for medical applications narrower wavelength band is used, from 8 to $12 \mu \mathrm{m}[3]$.

Measurement procedure is a combination of standardized methods of patch testing and medical thermography with addition of visual images registration. Patch test reading are performed after 48 and 96 hours since allergen's application. Data acquisition is made after patch test reading with standard adhering. First, while patient is acclimatizing, images in different ranges of light radiation are taken with the use of AV MAKO G-125C, then thermograms are captured with the use of FLIR A300 camera. Additionally, in the field of view of infrared camera a reference temperature module is visible.

Data acquisition was taken at the Medical Centre Med-All in Krakow, during standard patch test procedure. After changes in data acquisition procedure (in relation to [7]) tests were proceeded on group of 21 patients (18 females, 3 males, aged 26-77 years). All participants provided written informed consent.

Registration of infrared and visible light images at the same position and time, enables to precisely define in which location of the chamber the thermal positive skin reaction to allergen has occurred (Fig. 1). 


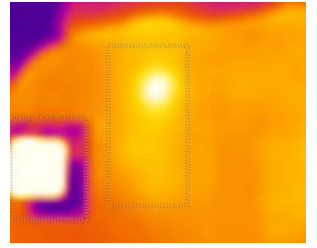

(a)

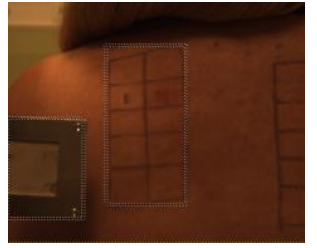

(b)

Fig. 1. Combining of images: (a) infrared; (b) visible light.

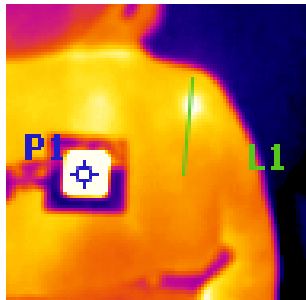

(a)

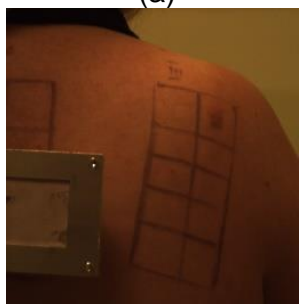

(c)

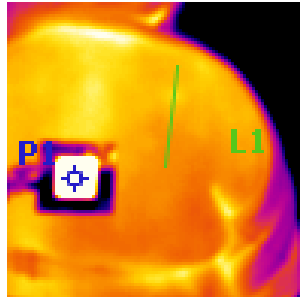

(b)

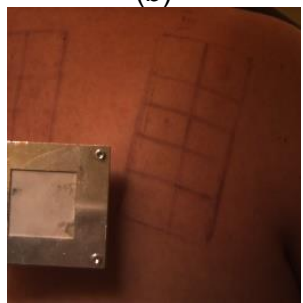

(d)

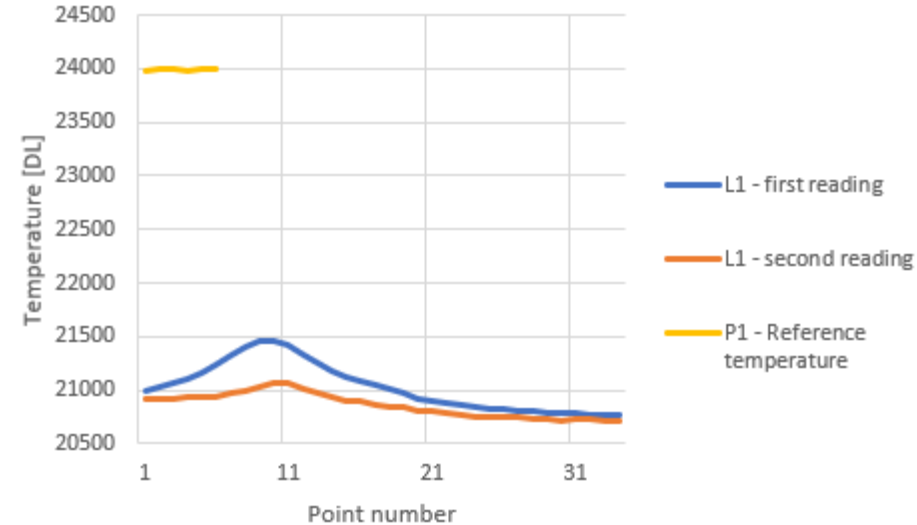

(e)

Fig. 2. Patient with strong reaction to 1 allergen. Thermal image of the skin after patch testing with L1 - line temperature analysis and P1 - reference temperature point: (a) Thermograph - first reading; (b) Thermograph - second reading; (c) Visual image -first reading; (d) Visual image - second reading; (e) Temperature changes (in digital units) at $L 1$ section at first reading (blue) and second reading (orange).

As it is shown at Fig. 2, temperature gradient at the skin area where positive reaction occurred are well visible. Such images can improve proper medical diagnosis, especially in cases when allergic reaction is weak or delayed.

The need for acclimatization is inconvenient for the patient and problematic for the medical centre, but necessary to obtain reliable results.

Proper definition of chamber on the skin with positive reaction to allergen is crucial, otherwise the wrong allergen might be indicated as the one for which the patient is allergic. Usage of reference temperature module makes possible to compare images acquired at different time by proper camera calibration and unification of the values of the histograms. For tests performed on patients it allows to check the difference in skin temperature at regions where patch tests were incubated in comparison to the neighbouring skin regions and reference temperature.

\section{REFERENCES}

[1] Fonacier L., Bernstein D., Pacheco K., et al., "Contact dermatitis: a practice parameter-Update 2015", Journal of Allergy and Clinical Immunology: In Practice, vol. 3 no. 3S, 2015.

(https://www.aaaai.org/Aaaai/media/MediaLibrary/PDF\%20Documents/Practice\%20and\%20Parameters/Contac t-dermatitis-2015.pdf)

[2] James A. P., Dasarathy B. V., "Medical Image Fusion: A survey of the state of the art", Information Fusion, vol. 19, pp. 4-19, 2014.

[3] Lahiri B.B., Bagavathiappan S., Jayakumar, Philip J., "Medical applications of infrared thermography: A review", Infrared Physics \& Technology, vol. 55, pp. 221-235, 2012.

[4] Hildebrandt C., Raschner C., Ammer K., "An Overview of Recent Application of Medical Infrared Thermography in Sports Medicine in Austria", Sensors, 10 (5), pp. 4700-4715, 2010.

[5] Szentkuti A., Kavanagh H.S., Grazio S., "Infrared thermography and image analysis for biomedical use", Periodicum biologorum, vol. 113, no 4, pp. 385-392, 2011.

[6] http://www.icdrg.org (accessed December 23, 2019)

[7] Szwedo M., Tomaka B., "Evaluation of patch tests results - research and development of technique based on infrared thermography", Diagnostyka, ISSN 1641-6414, vol. 16 no. 3, pp. 3-8, 2015. 